\section{Erhöhen Feinstaub und Verkehrsbelastung das PAH-Risiko?}

Sofianopoulou E et al. Traffic exposures, air pollution and outcomes in pulmonary arterial hypertension: A United Kingdom cohort study analysis. Eur Respir ] 2019; [Epub ahead of print] doi:10.1183/13993003.01429-2018

Weltweit werden 7 Millionen Todesfälle pro Jahr auf die Exposition mit Verkehr und Luftverschmutzung zurückgeführt. Immer neue Auswirkungen der Verkehrsbelastung und Luftverschmutzung auf die Gesundheit können nachgewiesen werden. Eine aktuelle Studie untersucht, ob auch eine Assoziation dieser Faktoren mit der pulmonalen arteriellen Hypertonie (PAH) besteht.

Die Arbeitsgruppe um Eleni Sofianopoulou von der Universität Cambridge untersuchte dazu die Exposition von $301 \mathrm{~Pa}$ tienten mit idiopathischer oder erblicher $\mathrm{PAH}$, die an der nationalen UK-PAH-Kohortenstudie teilnehmen. Die Forscher analysierten für alle Patienten die Exposition mit Feinstaub $\left(\leq 2,5 \mu \mathrm{m}^{3}, \mathrm{PM}_{2,5}\right)$, Stickstoffdioxid $\left(\mathrm{NO}_{2}\right)$ und durch indirekte Faktoren, die auf die verkehrsabhängige Luftverschmutzung schließen lassen, wie der Abstand zur Hauptstraße und die Länge der Straßen in der Pufferzone rund um die Wohnung der Patienten. Diese Ergebnisse setzten sie ins Verhältnis zum transplantationsfreien Überleben und der Schwere der pulmonalen Hämodynamik zu Studienbeginn.

\section{Ergebnisse}

Eine höhere geschätzte $\mathrm{PM}_{2,5}$-Expositon war assoziiert mit einem erhöhten Risiko für Tod oder Lungentransplantation. Nicht adjustiert ergab sich eine Hazard

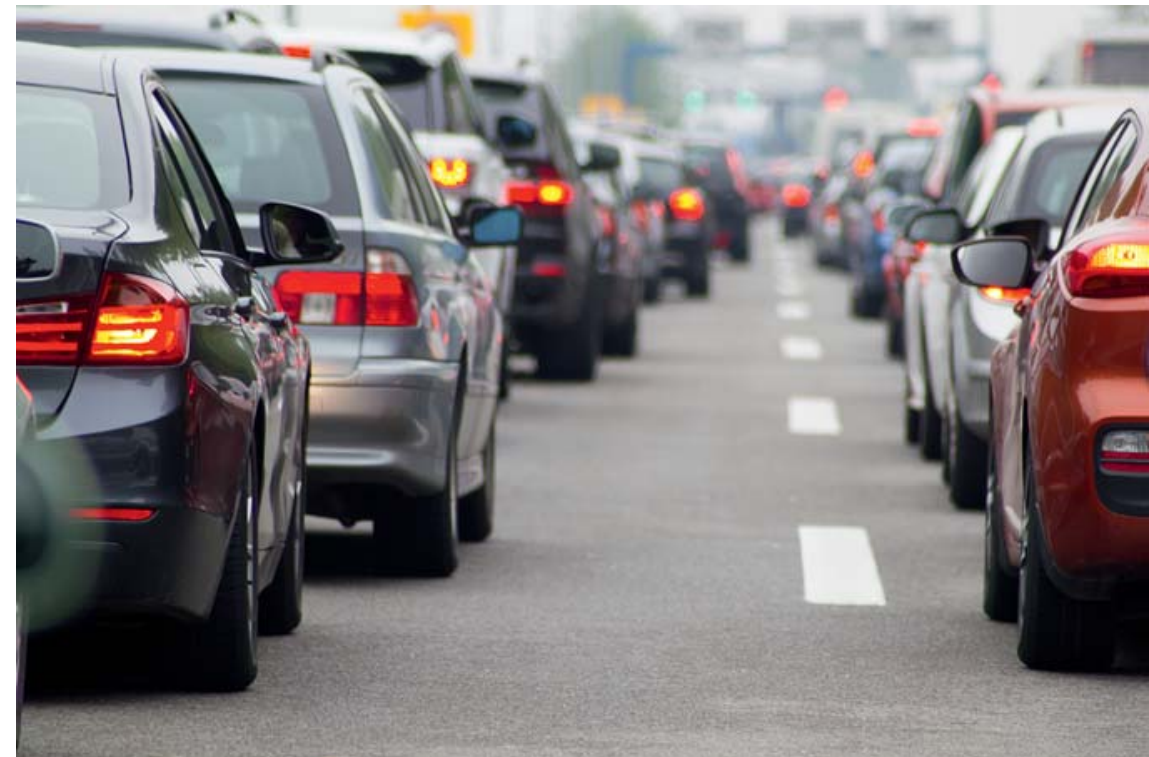

- In der britischen Studie wurden die Auswirkungen von verkehrsabhängiger Luftbelasung auf den Krankheitsverlauf bei PAH-Patienten untersucht. Quelle: disq - stock.adobe.com

Ratio (HR) von 2,68 pro $3 \mu \mathrm{PM}_{2,5} / \mathrm{m}^{3}$ mehr (95\% Konfidenzintervall [KI] 1,116,47; $p=0,028)$. Wurden andere vorab definierte Einflussfaktoren mit berücksichtigt, änderte das nichts an der signifikanten Assoziation (HR 4,38; $95 \% \mathrm{KI}$ $1,44-13,36$ pro $\left.3 \mu \mathrm{g} / \mathrm{m}^{3} ; \mathrm{p}=0,009\right)$.

Eine Assoziation von $\mathrm{NO}_{2}$-Exposition oder anderen verkehrsabhängigen Luftverschmutzungsparametern fand sich dagegen nicht.

Allerdings ergaben die indirekten Parameter für die verkehrsabhängige Luftbelastung innerhalb von 500 bis $1000 \mathrm{~m}$ rund um die Wohnadresse eine Korrelation mit den Risikokategorien der Europäischen Atemwegsgesellschaft ERS und der Europäischen kardiologischen Gesellschaft ESC und der pulmonalen Hämodynamik zu Studienbeginn. Diese Assoziation war am stärksten ausgeprägt beim vaskulären Widerstand in der Lunge.
FAZIT

In dieser Kohorte von Patienten mit idiopathischer oder erblicher PAH weisen indirekte Parameter auf einen Einfluss der verkehrsabhängigen Luftbelastung auf die Erkrankungsschwere bei Studieneinschluss hin, während die Feinstaubbelastung ein unabhängiger Prädiktor für ein kürzeres transplantatfreies Überleben zu sein scheint. Die Autoren räumen ein, dass diese Ergebnisse mit Vorsicht zu bewerten sind und weitere Studien zur Validierung und Bestätigung an größeren Kohorten notwendig sind.

Friederike Klein, München 